\title{
Unterrichtsqualität in der Religionsdidaktik - fachspezifische und fachübergreifende Aspekte
}

\author{
Martin Rothgangel $(\mathbb{D}$
}

Eingegangen: 17. Dezember 2020 / Überarbeitet: 24. März 2021 / Angenommen: 28. April 2021 / Online publiziert: 28. Mai 2021

(C) Der/die Autor(en) 2021

Zusammenfassung Von Ausnahmen abgesehen weist der religionsdidaktische Diskurs bislang v.a. zwei Problempunkte auf: Zwar wurden diverse religionsdidaktische „Qualitätsmodelle“ publiziert, jedoch nehmen diese oftmals unzureichend aufeinander Bezug und es dominiert der theoretische Diskurs über Qualität gegenüber der empirischen Überprüfung der entsprechenden Konzepte. Dementsprechend zeigt auch der Vergleich mit dem Syntheseframework von Praetorius et al. einerseits deutliche religionsdidaktische Forschungsdesiderate auf; andererseits weisen religionsdidaktische Qualitätsmodelle auf die Bedeutung von Kontextfaktoren des Unterrichts hin und werfen drei grundsätzliche Fragen auf, die generell für die bildungswissenschaftliche Bestimmung fachspezifischer und fachübergreifender Aspekte von Unterrichtsqualität relevant sein können.

Schlüsselwörter Qualitätsmodelle · Empirische Überprüfung · Guter Fachunterricht $\cdot$ Besonderes Profil

Martin Rothgangel $(\bowtie)$

Institute of Religious Education, University of Vienna, Wien, Österreich

E-Mail: martin.rothgangel@univie.ac.at 


\title{
Teaching quality in religious education - subject-specific and cross- disciplinary aspects
}

\begin{abstract}
Apart from a few exceptions, the discourse on religious education has two main problems so far: Although various "quality models" for religious education have been published, they often do not sufficiently refer to each other and the theoretical discourse on quality dominates over the empirical examination of the respective concepts. Accordingly, the comparison with the synthesis framework of Praetorius et al. on the one hand shows clear research desiderata of religious education; on the other hand, quality models of religious education point to the importance of contextual factors of teaching and raise three fundamental questions that can be generally relevant for the determination of subject-specific and interdisciplinary aspects of teaching quality.
\end{abstract}

Keywords Quality models · Empirical evaluation · Good subject teaching · Distinct profile

Überblickt man den religionsdidaktischen Diskurs zur Qualitätsfrage des Religionsunterrichts, dann lässt sich seit den empirischen Studien Anton Buchers zum Religionsunterricht in Österreich und Deutschland (1996, 2000) v. a. im Zeitraum zwischen 2000 und 2010 ein ausgesprochen lebendiger Dialog greifen (z. B. Nordhofen 2001; Rothgangel 2003; Jahrbuch für Religionspädagogik 2006; Pirner 2008), der sogar Eingang in evangelische wie katholische Lehrbücher findet (z. B. Adam und Rothgangel 2012; Bahr 2013). Die religionsdidaktische Diskussion über Unterrichtsqualität verflacht zwar in der Folgezeit, die Qualitätsfrage steht jedoch in zwei aktuellen religionspädagogischen Monographien erneut im Zentrum (Schwarz 2019, v. a. S. 543-602; Schweitzer 2020) und soll - was die Bedeutung von Schweitzers Monographie unterstreicht - im Projekt QUIRU (,Qualität und Qualitätsentwicklung im Religionsunterricht") auf breiter Basis untersucht werden (Schweitzer 2020, S. 21, 204-208; www.uni-tuebingen.de/quiru). Von Ausnahmen abgesehen weist der religionsdidaktische Diskurs bislang v. a. zwei Problempunkte auf: Zwar wurden diverse religionsdidaktische „Qualitätsmodelle“ publiziert (z. B. Bucher 2000; Schweitzer 2006; Englert 2006; Hanisch 2012; Adam und Rothgangel 2012), jedoch nehmen diese oftmals unzureichend aufeinander Bezug (Schwarz 2019, S. 566-568, 603 ) und es dominiert der theoretische Diskurs über Qualität gegenüber der empirischen Überprüfung der entsprechenden Konzepte (Schwarz 2019, S. 603; Schweitzer 2020, S. 36f.).

\section{Die Diskussion der Fachspezifität von Unterrichtsqualität in der Religionsdidaktik}

Ungeachtet aller Unterschiede im Detail ist es in der religionsdidaktischen Diskussion unstrittig, dass die religionsunterrichtliche Qualität zugleich von fachübergreifenden und fachspezifischen Aspekten geprägt ist. Am ausführlichsten findet sich 
eine entsprechende Reflexion bei Schweitzer (2020, S. 38-58), der treffend zwischen den folgenden drei Bestimmungshorizonten von Qualität unterscheidet, „die den Religionsunterricht jeweils in eine andere Perspektive rücken: guter Unterricht", ,guter Fachunterricht" sowie das besondere Profil des Religionsunterrichts" (ebd., S. 38), wobei die letztgenannte Perspektive bestimmte Fachspezifika des Religionsunterrichts besonders hervorhebt. In der ersten Bestimmungsperspektive des ,guten Unterrichts` werden die berechtigen Aspekte fachübergreifender Qualitätskriterien u. a. damit begründet, dass für den Religionsunterricht an öffentlichen Schulen grundsätzlich die gleichen Kriterien wie für alle anderen Fächer gelten (ebd., S. 39). Dementsprechend sind für den Religionsunterricht die in der empirischen Bildungsforschung sowie in der Schulpädagogik und Allgemeinen Didaktik formulierten Erkenntnisse von gutem Unterricht von hoher Relevanz und es erfolgt z. B. bei Schwarz (2019, S. 577-605) und Schweitzer (2020, S. 117-119) u. a. eine Rezeption des ,Angebots-Nutzungs-Modells“ von Helmke.

Für den zweiten Bestimmungshorizont ,guter Fachunterricht“ sind im vorliegenden Fall des Religionsunterrichts zwei Faktoren maßgeblich: Erstens fungieren für den konfessionellen Religionsunterricht die Theologie sowie für die ,neutrale ' Religionskunde die Religionswissenschaft als primäre Bezugswissenschaften, die wiederum ganz bestimmte und durchaus verschiedene fachliche Ziele und Inhalte implizieren. Zweitens ist die Religionsdidaktik für die fachliche Zielbestimmung sowie für die notwendige Auswahl theologischer bzw. religiös relevanter Themen in Curricula eine entscheidende Reflexionsinstanz. Beide Faktoren führen gemeinsam zu spezifischen Kompetenzen religiöser Bildung, nach denen die fachspezifische Qualität des Religionsunterrichts zu beurteilen ist (ebd., S. 48, 51). Beispielhaft lassen sich in dieser Hinsicht die von den katholischen Bischöfen erlassenen Kompetenzen für den Religionsunterricht der Sekundarstufe I anführen, die auf religionsdidaktischer Expertise basieren: „religiöse Phänomene wahrnehmen“, ,religiöse Sprache verstehen und verwenden“, ,religiöse Zeugnisse verstehen“; ,religiöses Wissen darstellen“, ,,in religiösen Fragen begründet urteilen“, ,,sich über religiöse Fragen und Überzeugungen verständigen“ und ,aus religiöser Motivation handeln“ (Deutsche Bischofskonferenz 2004, S. 16).

Der Hinweis auf eine Verlautbarung deutscher Bischöfe zum Religionsunterricht ist bewusst gewählt, weil dies eine Besonderheit des Religionsunterrichts dokumentiert und unmittelbar überleitet zum dritten Bestimmungshorizont, dem spezifischen Profil des Religionsunterrichts. Dieses lässt sich in dreifacher Weise entfalten (Schweitzer 2020, S. 52-56): Erstens sind aufgrund der Neutralität des Staates hinsichtlich religiöser Fragen die Inhalte der konfessionellen Religionsunterrichte in Übereinstimmung mit den jeweiligen Religionsgemeinschaften zu bestimmen, was konkret deren Mitwirkung bei Lehrplänen und Schulbüchern betrifft und damit auch fachspezifische Qualitätskriterien des Religionsunterrichts. Zweitens dokumentieren diverse empirische Studien, dass die persönlichen Glaubensüberzeugungen von Religionslehrer*innen eine wichtige Rolle für religiöse Lehr- und Lernprozesse spielen (z. B. Schwarz 2019, S. 604). Sie müssen, ,sich hier fragen lassen, ,ob sie denn das selber glauben, was sie da sagen“" (Schweitzer 2020, S. 54). Darüber hinaus werden immer auch seelsorgerliche Kompetenzen und ein persönliches Ethos von Religionslehrer*innen erwartet, für die es in anderen Fächern auf diese Weise gleichfalls 
Tab. 1 Qualitätsdimensionen nach Schwarz (2019) und Praetorius et al. (2020)

\begin{tabular}{|c|c|}
\hline Übersicht Religionsdidaktik Schwarz (2019) & Syntheseframework Praetorius et al. (2020) \\
\hline $\begin{array}{l}\text { 1. Strukturqualität (z. B. organisatorische Rahmen- } \\
\text { bedingungen wie ausreichende Stundenzahl, kein } \\
\text { Randstundenfach usw.) }\end{array}$ & - \\
\hline $\begin{array}{l}\text { 2. Prozessqualität (z. B. Ermöglichung von Selbsttätig- } \\
\text { keit, SuS-Orientierung; Klassenführung; Umgang mit } \\
\text { Heterogenität) }\end{array}$ & $\begin{array}{l}\text { Vgl. 2. Kognitive Aktivierung } \\
\text { vgl. 5. Unterstützung des Lernens aller Schü- } \\
\text { ler*innen } \\
\text { vgl. 7. Klassenführung }\end{array}$ \\
\hline $\begin{array}{l}\text { 3. Fachwissenschaft-Themen (z.B. theologisch ver- } \\
\text { antwortet; Brücken zwischen den Themen und den } \\
\text { Erfahrungen der Schüler*innen) }\end{array}$ & $\begin{array}{l}\text { Vgl. 1. Auswahl und Thematisierung von } \\
\text { Inhalten und Fachmethoden }\end{array}$ \\
\hline $\begin{array}{l}\text { 4. Beziehungsqualität/Atmosphäre (z. B. Wertschät- } \\
\text { zung) }\end{array}$ & Vgl. 6. Sozio-emotionale Unterstützung \\
\hline $\begin{array}{l}\text { 5. Produkt-/Ergebnisqualität (z. B. Konsolidierung und } \\
\text { Sicherung) }\end{array}$ & Vgl. 3. Unterstützung des Übens \\
\hline 6. Konzeptqualität (z. B. konzeptionelle Klarheit) & - \\
\hline 7. Reflexion - Evaluation (z.B. Metaunterricht) & Vgl. 4. Formatives Assessment \\
\hline 8. Systemqualität (z. B. Beitrag zum Schulleben) & - \\
\hline
\end{tabular}

kaum eine Entsprechung gibt. Drittens spielen generell im Religionsunterricht existenzielle Bezüge eine herausragende Rolle, da im Religionsunterricht all das, was für Menschen von existenzieller Bedeutsamkeit sein kann (z. B. Geld, Musik, Sport) ein Gegenstand des Religionsunterrichts ist. Dementsprechend ist der Religionsunterricht vermutlich das einzige Unterrichtsfach, wo das mit der didaktischen Analyse vergleichbare Elementarisierungsmodell eine Dimension enthält, die ,elementare Wahrheiten“" lautet (Schweitzer 2020, S. 50, 53, 163-167).

Vor dem Hintergrund des zweiten und dritten Bestimmungshorizonts (,guter Fachunterricht", ,,besonderes Profil des Religionsunterrichts“) überrascht es schließlich nicht, dass zwar das „Angebots-Nutzungs-Modell“ von Helmke rezipiert wird, jedoch z.B. von Schweitzer (2020, S. 118) fachspezifische Modifikationen vorgenommen werden: Er ergänzt das Feld „Unterricht“ (=Angebot) um die aus religionsdidaktischer Perspektive wesentliche Förderung von Wissen, Verstehen, Perspektivenübernahme und Toleranz (ebd., S. 118, 74-105). Darüber hinaus ermittelt Schwarz (2019, S. 566-568) anhand des Vergleichs von sechs religionsdidaktischen Qualitätsmodellen folgende acht Qualitätsdimensionen, wobei in Tab. 1 die Dimensionen des Syntheseframeworks von Praetorius et al. (2020) zum Vergleich herangezogen werden.

Zunächst wird anhand dieser Übersicht deutlich, dass zumindest zwei religionsdidaktische Qualitätsdimensionen deutlich fachspezifisch geprägt sind: „Fachwissenschaft-Themen“ sowie „Konzeptqualität“. Allerdings ist das Verständnis von Konzeptqualität in den sechs religionsdidaktischen Qualitätsmodellen, die von Schwarz herangezogen werden, sehr heterogen: Beispielsweise wird diesbezüglich nicht nur „konzeptionelle Klarheit“" genannt, sondern können auch „fachdidaktische Planung“, „grundlagentheoretische Verortung“ oder „Unterscheidung zwischen unverfügbaren Zielen und operationalisierbaren Bildungsstandards“ (ebd., S. 657) angeführt werden. Bedingt durch die Zusammenstellung von sechs religionsdidaktischen Qua- 
litätsmodellen und deren Verschiedenheit findet sich eine vergleichbare Disparität auch bei anderen Subdimensionen bzw. Indikatoren. Aus diesem Grund ist der vorliegende Vergleich mit Vorbehalt zu betrachten. Grundsätzlich ist das Syntheseframework von Praetorius u. a. mit seinen Dimensionen, Subdimensionen und Indikatoren weitaus präziser und konsistenter. Gleichwohl könnte vor dem Hintergrund der Übersicht von Schwarz (2019) bedacht werden, ob nicht Kontextfaktoren des Unterrichts (vgl. Struktur- und Systemqualität) sowie die Frage nach den fachlichen und methodischen Grenzen der Messung von Unterrichtsqualität (vgl. Konzeptqualität) zu ergänzen sind. Gerade in „,kleinen“ Fächern mit problematischen curricularen Rahmenbedingungen und einer Randlage in der Stundentafel tritt die Bedeutung von Kontextfaktoren für die Qualität des Fachunterrichts immer wieder hervor.

Abschließend sei angemerkt, dass die vorliegende Darstellung spezifisch für den deutschsprachigen Bereich ist und sich im internationalen Kontext entweder durch eine stärkere religionswissenschaftliche Orientierung (z. B. England) oder umgekehrt durch eine stärkere katechetische Prägung (z.B. Polen) unterschiedliche Verständnisweisen von der Qualität des Religionsunterrichts bestehen können.

\section{Eigene Positionierung zur Frage der Fachspezifität von Unterrichtsqualität}

Trotz vermehrter empirischer Studien in der Religionsdidaktik, die explizit oder implizit für den Qualitätsdiskurs relevant sind (Schweitzer 2020, S. 123-149; Schwarz 2019), ist die Forschung in diesem Bereich noch nicht so weit vorangeschritten, dass der Verfasser evidenzbasiert ein begründetes Urteil abgeben könnte, wie auf der Ebene von Dimensionen, Subdimensionen und Indikatoren (Praetorius et al. 2020) religionsspezifische und fachübergreifende Aspekte zueinanderstehen.

Jedoch treten durch den religionsdidaktischen Qualitätsdiskurs drei grundsätzliche Fragen hervor, die m. E. generell für die bildungswissenschaftliche Bestimmung fachspezifischer und fachübergreifender Aspekte von Unterrichtsqualität relevant sein könnten:

\subsection{Was sind die Grenzen von Qualitätskriterien und worin besteht ihre Bedeutung?}

Der Religionsunterricht besitzt aufgrund seines Bezugs auf Transzendenz, d.h. auf den Bereich jenseits menschlicher Sinneswahrnehmungen und möglicher Erfahrungen, notwendig auch fachspezifische Grenzen der Messung von Unterrichtsqualität (vgl. auch Schweitzer 2020, S. 56f.). Diese Grenze zwischen Operationalisierbarkeit und Nicht-Operationalisierbarkeit verläuft in den Unterrichtsfächern keineswegs einheitlich, sondern ist fachlich bedingt: „Natur“ kann aus naturwissenschaftlicher Perspektive mittels intersubjektiv nachvollziehbarer Experimente erforscht werden, die theologische Reflexion über „Schöpfung“ setzt hingegen das Vertrauen auf das Schöpfungshandeln Gottes voraus. Die notwendige und wichtige Frage nach den Qualitätskriterien guten Fachunterrichts schließt somit auch die Reflexion über die Grenzen dieser Modelle und Kriterien von Qualität ein - sei dies methodologisch 
oder fachspezifisch begründet. Der mögliche Ertrag dieser Überlegungen besteht darin, dass sich bildungswissenschaftliche Forschung und bildungspolitische Weichenstellungen nicht zu einseitig auf die operationalisierbaren Aspekte des Fachunterrichts fokussieren und für den Bildungsprozess von Kindern und Jugendlichen gleichermaßen wichtige nicht-operationalisierbare Aspekte vernachlässigen. Die Frage nach der Qualität guten (Fach-)Unterrichts sollte somit auch die grundlagentheoretische Frage nach den Grenzen von Qualitätsbestimmungen bewusst halten, damit Schule und Unterricht sich nicht unversehens auf Messbares kaprizieren und verengen.

\subsection{Welche Akteure bestimmen Modelle von Unterrichtsqualität?}

Als ein religionsdidaktisches Spezifikum ist hervorgetreten, dass neben ExpertInnen aus den Bildungswissenschaften, der Theologie sowie der Religionsdidaktik auch die Religionsgemeinschaften ein Akteur sind, die in fachlicher Hinsicht über die Qualität des Religionsunterrichts mitbestimmen. Darüber hinaus ist zu bedenken, inwieweit auch die Erfahrungen von Religionslehrkräften und sogar Schüler*innen einzubeziehen sind, um ein praxisrelevantes Verständnis von Unterrichtsqualität zu gewinnen. In Anbetracht des (theologisch in der Gottebenbildlichkeit des Menschen begründeten) religionsdidaktischen Prinzips der Subjektorientierung und im Sinne einer Theologie von Kindern und Jugendlichen ist im Religionsunterricht deren Reflexion ihres (Lebens-)Glaubens nicht einfach als ,,minderwertiger“ Ausgangszustand zu beurteilen. Vielmehr weht, bildlich gesprochen, der Heilige Geist wo er will, und sind aus religionsdidaktischer Perspektive auch religiös relevante Reflexionen jenseits von ExpertInnen aus Theologie und Klerus ernst zu nehmen (Rothgangel 2010). Nicht zuletzt zeigt auch die Bezugnahme auf bildungswissenschaftliche Forschung, dass die Einschätzungen von Schüler*nnen zwar nicht ,unhinterfragt als Positivkriterium“ (Schwarz 2019, S. 543) heranzuziehen sind - gleichwohl ist das Verständnis eines guten Religionsunterrichts ,nicht an den AdressatInnen religiöser Bildungsprozesse vorbei zu entwerfen“ (ebd., S. 543). Dieser Punkt ist auch hinsichtlich der Gestaltungsformen des Religionsunterrichts zu bedenken, da ein konfessioneller Religionsunterricht, der strikt getrennt durchgeführt wird, andere Qualitätskriterien impliziert als ein Religionsunterricht, bei dem eine interkonfessionelle Kooperation stattfindet.

Schließlich wirkt sich die Reflexion nach den Akteuren, welche bei der Bestimmung von Unterrichtsqualität einzubeziehen sind, gleichfalls auf das Verhältnis von fachspezifischen sowie fachübergreifenden Aspekten aus, wie ein einfaches Gedankenspiel zeigt: Die Einbeziehung von VertreterInnen aus der Theologie führt wahrscheinlich zu fachspezifischen, die von VertreterInnen aus der Allgemeinen Didaktik eher zu fachübergreifenden Akzentuierungen.

\subsection{Welches Prozedere gewährleistet eine adäquate Bestimmung von Unterrichtsqualität?}

Nicht nur aus religionsdidaktischer, sondern generell aus fachdidaktischer Perspektive ist m. E. folgender Punkt hervorzuheben: Qualitätsmodelle aus empirischer Bil- 
dungsforschung und Allgemeiner Didaktik nehmen oftmals unzureichend im Sinne einer induktiven Denkbewegung fachspezifische Erkenntnisse aus verschiedenen Fachdidaktiken auf, um diese konstitutiv für ihr Modell zu berücksichtigen. Die Vorgehensweise des vorliegenden Themenheftes ist demgegenüber mustergültig und wegweisend für die bildungswissenschaftliche Erforschung fachlichen Unterrichtens, da sie - entsprechend der Logik einer Allgemeiner Fachdidaktik (Rothgangel et al. 2020) - gezielt Top-Down und Bottom-Up Überlegungen miteinander verschränkt. Qualitätsmodelle wie die von Helmke (2017) oder Praetorius et al. (2020) sind im Sinne evidenzbasierter Wissenschaft an verschiedenen (!) konkreten Unterrichtsfächern zu überprüfen und müssen gegebenenfalls ergänzt, korrigiert oder im Extremfall komplett revidiert werden. Die bildungswissenschaftliche Qualitätsforschung wird zwar komplexer, wenn sie konsequent die verschiedenen Unterrichtsfächer und ihre Fachdidaktiken berücksichtigt, v. a. auch diejenigen, die nicht vom Leibniz-Institut für die Pädagogik der Naturwissenschaften und Mathematik (IPN) sowie vom Institut zur Qualitätsentwicklung im Bildungswesen (IQB) beforscht werden. Aber nur auf diese Weise ist eine optimale Passung von Dimensionen, Subdimensionen sowie Indikatoren von Unterrichtsqualität einerseits mit der faktisch vorhandenen Vielzahl an schulischen Unterrichtsfächern andererseits zu erzielen.

Funding Open access funding provided by University of Vienna.

Open Access Dieser Artikel wird unter der Creative Commons Namensnennung 4.0 International Lizenz veröffentlicht, welche die Nutzung, Vervielfältigung, Bearbeitung, Verbreitung und Wiedergabe in jeglichem Medium und Format erlaubt, sofern Sie den/die ursprünglichen Autor(en) und die Quelle ordnungsgemäß nennen, einen Link zur Creative Commons Lizenz beifügen und angeben, ob Änderungen vorgenommen wurden.

Die in diesem Artikel enthaltenen Bilder und sonstiges Drittmaterial unterliegen ebenfalls der genannten Creative Commons Lizenz, sofern sich aus der Abbildungslegende nichts anderes ergibt. Sofern das betreffende Material nicht unter der genannten Creative Commons Lizenz steht und die betreffende Handlung nicht nach gesetzlichen Vorschriften erlaubt ist, ist für die oben aufgeführten Weiterverwendungen des Materials die Einwilligung des jeweiligen Rechteinhabers einzuholen.

Weitere Details zur Lizenz entnehmen Sie bitte der Lizenzinformation auf http://creativecommons.org/ licenses/by/4.0/deed.de.

\section{Literatur}

Adam, G., \& Rothgangel, M. (2012). Was ist guter Religionsunterricht? In M. Rothgangel, G. Adam \& R. Lachmann (Hrsg.), Religionspädagogisches Kompendium (7. Aufl. S. 416-433). Göttingen: V\&R. Bahr, M. (2013). Guten Religionsunterricht in den Blick nehmen. In G. Hilger, S. Leimgruber \& H.G. Ziebertz (Hrsg.), Religionsdidaktik. Ein Leitfaden für Studium, Ausbildung und Beruf (6. Aufl. S. 487-497). München: Kösel.

Bucher, A. A. (1996). Religionsunterricht: Besser als sein Ruf? Empirische Einblicke in ein umstrittenes Fach. Innsbruck: Tyrolia.

Bucher, A. A. (2000). Religionsunterricht zwischen Lernfach und Lebenshilfe. Eine empirische Untersuchung zum katholischen Religionsunterricht in der Bundesrepublik Deutschland. Stuttgart: Kohlhammer.

Deutsche Bischofskonferenz (Hrsg.). (2004). Kirchliche Richtlinien zu Bildungsstandards für den Katholischen Religionsunterricht in den Jahrgangsstufen 5-10/Sekundarstufe I. Bonn: Sekretariat der Deutsche Bischofskonferenz. 
Englert, R. (2006). Die Diskussion um Unterrichtsqualität - und was die Religionsdidaktik daraus lernen könnte. Jahrbuch für Religionspädagogik, 22, 52-64.

Hanisch, H. (2012). Was ist guter Religionsunterricht? In H. Hanisch \& Chr Gramzow (Hrsg.), Religionsunterricht im Freistaat Sachsen. Lernen, Lehren und Forschen seit 20 Jahren. Leipzig: EVA.

Helmke, A. (2017). Unterrichtsqualität und Lehrerprofessionalität. Diagnose, Evaluation und Verbesserung des Unterrichts (7. Aufl.). Selze-Velber: Klett.

Jahrbuch für Religionspädagogik (2006). Was ist guter Fachunterricht? Hrsg. v. C. Bizer u.a. Neukirchen: Neukirchener Verlag.

Nordhofen, E. (2001). Unbefriedigender und unbefriedigter Religionsunterricht. Anmerkungen zu einer fruchtbaren Debatte. Engagement, 19(1):23-36.

Pirner, M.L. (2008). Auf der Suche nach dem guten Religionsunterricht. Perspektiven religionsdidaktischer Lehr-Lern-Forschung. In Religionspädagogische Beiträge 60 (S. 3-17).

Praetorius, A.-K., Herrmann, Ch, Gerlach, E., Zülsdorf-Kersting, M., Heinitz, B., \& Nehring, A. (2020). Unterrichtsqualität in den Fachdidaktiken im deutschsprachigen Raum - zwischen Generik und Fachspezifität. Unterrichtswissenschaft: Zeitschrift für Lernforschung.. https://doi.org/10.1007/s42010020-00082-8.

Rothgangel, M. (2003). Qualitätskriterien ,guten“ Religionsunterrichts. In D. Fischer \& M. Rothgangel (Hrsg.), Standards für religiöse Bildung. Zur Reformdiskussion in Schule und Lehrerbildung (S. 104-118). Münster: LIT.

Rothgangel, M. (2010). „Die immer für mich da sind“ - Familie und Freunde als Heilige des Alltags. In M. Rothgangel \& H. Schwarz (Hrsg.), Götter, Heilige, Heroen (S. 205-224). Frankfurt: Peter Lang.

Rothgangel, M., Abraham, U., Bayrhuber, H., Frederking, V., Jank, W., \& Vollmer, H. J. (Hrsg.). (2020). Lernen im Fach und über das Fach hinaus. Bestandsaufnahmen und Forschungsperspektiven aus 17 Fachdidaktiken im Vergleich. Allgemeine Fachdidaktik, Bd. 2. Münster, New York: Waxmann.

Schwarz, S. (2019). SchülerInnenperspektiven und Religionsunterricht. Empirische Einblicke - Theoretische Überlegungen. Stuttgart: Kohlhammer.

Schweitzer, F. (2006). „Guter Religionsunterricht“ - aus der Sicht der Fachdidaktik. Jahrbuch für Religionspädagogik, 22, 41-51.

Schweitzer, F. (2020). Religion noch besser unterrichten. Qualität und Qualitätsentwicklung im RU. Göttingen: V\&R. 